A N N A L E S Annales de Bretagne et des Pays de l'Ouest

\title{
Dictionnaire des lycées catholiques de Bretagne
}

Jean-Luc Marais

\section{(2) OpenEdition}

Journals

Édition électronique

URL : https://journals.openedition.org/abpo/4290

DOI : $10.4000 /$ abpo.4290

ISBN : 978-2-7535-7720-6

ISSN : 2108-6443

Éditeur

Presses universitaires de Rennes

Édition imprimée

Date de publication : 18 décembre 2018

Pagination : 191-193

ISBN : 978-2-7535-7718-3

ISSN : 0399-0826

Référence électronique

Jean-Luc Marais, "Dictionnaire des lycées catholiques de Bretagne », Annales de Bretagne et des Pays de l'Ouest [En ligne], 125-4 | 2018, mis en ligne le 18 décembre 2018, consulté le 03 mars 2022. URL: http://journals.openedition.org/abpo/4290 ; DOI : https://doi.org/10.4000/abpo.4290 
geoise, qui lui permet de s'investir dans des œuvres sociales et culturelles. Cette bibliophile fut aussi secrétaire de la revue littéraire La Pensée bretonne, entre 1914 et 1920 , et mit sur pieds la bibliothèque de Plancoët, qu'elle anima bénévolement jusqu'à sa quasi-cécité, survenue dans les années 1950. Marie Le Gac-Salonne est également une artiste émérite, qui aime broder, créer et puiser son inspiration dans l'observation de la nature. C'est donc une personnalité éclectique et talentueuse qui émerge au fil des pages.

Isabelle Le Boulanger en brosse un portrait sensible, avec des mots justes. Sans fard ni pesanteur, elle amène le lecteur de la chambre à coucher aux salles de réunion et lui permet de comprendre les douleurs et les insatisfactions de Marie Le Gac-Salonne, en étayant son récit de nombreuses citations tirées de sa correspondance et de son journal intime. Ce livre est donc aussi une contribution à l'histoire de la vie privée de la bourgeoisie bretonne du début du xxe siècle, qui montre que l'acquisition d'une automobile De Dion-Bouton en 1909 ou d'une résidence secondaire à Saint-Cast en 1922 rapproche temporairement un couple désuni. La clarté du plan et la rigueur des introductions et des conclusions partielles rendent le propos limpide. On aurait toutefois aimé que ces dernières quittent parfois le terrain biographique pour mieux inscrire l'ouvrage dans l'historiographie et souligner ce qu'il apportait à la connaissance du féminisme ou de la Bretagne contemporaine. Le destin de Marie Le Gac-Salonne est aussi celui d'une bourgeoise morlaisienne déracinée en haute Bretagne, dont le féminisme réformiste fait une audacieuse synthèse entre l'héritage conservateur de sa mère et la part libérale léguée par son père. Les Ursulines et l'école laïque en somme.

La question de la transmission culturelle à l'intérieur de la famille, évoquée dans le premier chapitre tourné vers les générations antérieures, ressurgit en aval, dans un passionnant épilogue qui contient tous les ingrédients de Mères, filles. Sept générations, la stimulante saga de Juliet Nicholson. On y voit notamment le désir d'affirmation individuelle emprunter la voie religieuse. Alors que Marie Le GacSalonne avait pris ses distances avec un catholicisme qui entrait en conflit avec son projet de société, une de ses filles fit le chemin inverse en 1936. Bien plus, son unique petite-fille, Viviane Duvignac, entra chez les Sœurs de la Divine Providence de Créhen à l'âge de 25 ans, en 1950. C'est elle qui recueillit puis légua ses archives, en 1999. "Elle a respecté ma vocation comme son mari avait respecté son "apostolat féministe" ", affirme-t-elle. Son mari? Ses affaires furent aussi florissantes que son mariage morne : Henri Salonne se mua en entrepreneur, jouant un rôle pionnier dans la commercialisation de l'eau de Plancoët et le développement de la station balnéaire des Sables-d'Or. Il attend son historien, lequel trouvera dans les archives de Marie Le Gac-Salonne un magnifique éclairage sur sa vie privée.

Samuel GiCQUEL

Celton, Yann, Gicquel, Samuel, Le Moigne, Frédéric et Tranvouez, Yvon (dir.), Dictionnaire des lycées catholiques de Bretagne, PUR, 2018, 656 p.

Après le Dictionnaire des Lycées publics des Pays de la Loire (2009) réalisé à partir d'une idée d'Alain Croix, après le Dictionnaire des lycées publics de Bretagne (2012), voici le Dictionnaire des Lycées catholiques de Bretagne, qui mérite de retenir l'attention tant l'histoire de l'enseignement catholique est mal connue. Les trois textes introductifs (liminaire, préface, avant-propos) au contenu très conventionnel, peuvent inquiéter le lecteur, mais l'article Vocabulaire apporte (tardivement en raison de l'ordre alphabétique) une mise au point bienvenue, qui n'est pas que 
lexicale. Suit une riche introduction, qui démêle l'écheveau des notions de privé, de catholique, d'État et d'Église, de l'Ancien Régime à nos jours. Vient alors le corps de l'ouvrage : 109 notices d'établissements, 113 notices thématiques.

Les notices d'établissement de longueur variée (inégalité assumée par les directeurs de l'ouvrage) présentent des histoires que l'on peut qualifier de classiques. Au départ, des institutions assurant toutes les étapes de la scolarité, séparant garçons et filles; à l'arrivée, des établissements mixtes, accueillant le second cycle de l'enseignement secondaire et éventuellement des classes préparatoires. Mais un grand nombre d'établissements ont des parcours beaucoup plus sinueux, dont on ne peut dire (faute d'éléments de comparaison) s'il s'agit d'une originalité de la Bretagne ou de l'enseignement catholique. Nés d'une initiative locale généralement très modeste, parfois abrités dans une école primaire existante, des formations à caractère social, plutôt pour les filles, ou professionnel (agriculture, métiers liés à la mer) pour les garçons, toutes très liées à un territoire, se développent modestement, reçoivent une reconnaissance tardive par l'accès à un diplôme d'État, et se transforment en lycées agricoles ou professionnels, avec en général des regroupements et le maintien d'un ancrage local. Les notices d'établissements déroulent généralement une histoire heureuse, surmontant les crises politico-religieuses (interdiction de l'enseignement congréganiste), les guerres (destruction des locaux, occupation par l'ennemi), les évènements de 1968, pour aboutir aux établissements actuels, rayonnant de la modernité des bâtiments (clairement soulignée par l'illustration), de l'ouverture internationale, du dynamisme sur le marché de l'emploi. Originalité de l'enseignement catholique : le rôle de personnalités exceptionnelles, marquant fortement et longuement l'établissement qu'elles dirigent. Autre originalité, qui n'est sans doute pas sans rapport avec la précédente, la mention de crises graves (Petit Séminaire de Quintin, 1966; Le Paraclet à Quimper, 1971-1977; La Salle à Rennes, 1975-1976; Notre-Dame-de-la-Paix à Ploemeur, 1996).

Le regroupement des articles par lieux met en évidence (ce qui n'était pas le cas dans les précédents Dictionnaires) deux points importants. Le premier, bien connu, valant aussi bien pour le réseau public que le réseau privé, est la spécificité locale de chaque établissement, par le choix des filières proposées et par les caractéristiques sociales du recrutement. Le second est beaucoup plus important : la concurrence. Il s'agit ici bien sûr de la concurrence entre enseignement catholique et enseignement public (voir l'article Dualité et concurrence), mais aussi, moins connue à l'extérieur, la concurrence entre établissements catholiques. Alors que l'enseignement public est organisé verticalement et que sa stratégie est impulsée d'en haut, dans l'enseignement catholique tout part de l'établissement. Chaque établissement est indépendant : l'autorité diocésaine ne s'est imposée que tardivement, et doit composer avec l'autorité des congrégations enseignantes, des conseils d'administrations, des anciens élèves, et avec le poids des professeurs très attachés à leur maison. L'autonomie des établissements est renforcée par la loi Debré (1959), puisque c'est avec eux que l'État passe contrat. Mais cette indépendance des établissements n'est pas sans risque : les établissements peuvent être petits (ils sont en Bretagne plus petits que dans le reste de la France), menacés sur le "marché concurrentiel de l'offre d'enseignement " (p. 372). Le terme concurrence revient fréquemment dans le texte, et plusieurs articles l'évoquent (Élèves, Congrégations enseignantes, Parents d'élèves, Pédagogie, Regroupements d'établissements). Les perspectives de concentration (en particulier en ville) sont une réalité, et la Communication y joue un rôle essentiel : ce n'est pas sans raison qu'un article y est consacré.

Ce dictionnaire n'est pas seulement un dictionnaire. Par son introduction et par les articles thématiques, il déborde le cadre régional (Bretagne) et apporte des informations et une réflexion non conventionnelle sur l'enseignement catholique en France. Une série d'articles, rédigés principalement par Bruno Poucet, fait un 
tableau de l'histoire et de l'organisation de cet enseignement en France depuis 1959. Des renvois d'article à article auraient ici été utiles. Relevons les articles Défense scolaire, Caractère propre, Financement, Loi scolaire, Coût des études, Conseil régional, Immobilier, Organisation de l'enseignement catholique, Professeurs, Recrutement des personnel, etc. Une autre série (souvent sous la plume d'Yvon Tranvouez) aborde les déclinaisons de l'adjectif " catholique " accolé aux établissements. Là encore les enjeux abordés sont nationaux, et parfois même mondiaux, dans les articles Religion, Signes religieux, Aumônerie, Doctrine scolaire de l'Église, Religieuses, Vocations, Chrétiens contre l'école libre.

Terminons en soulignant quelques articles qui retiennent l'attention : les articles Jeunes filles et Mixité, les articles très originaux: Photo de classe, Parcs et jardins, Santé, Surnoms. L'article Mille-neuf-cent-quatre-vingt-quatre étonne (qu'est-ce donc que cette date?), mais éclaire le lecteur " extérieur " sur la sensibilité de l'enseignement catholique. L'historien appréciera les articles rétrospectifs Collèges d'Ancien Régime, ou Zouaves pontificaux, et les articles Établissements disparus, Juvénats, Petit séminaire (ici un renvoi aux articles sur chaque petit séminaire eut été utile). Si les personnels non enseignants actuels sont évoqués (Personnel Ogec), leurs prédécesseurs restent mal connus (quelques lignes impressionnantes dans l'article Religieuses). Même situation pour les professeurs : les prêtres diocésains professeurs ont peu attiré l'attention des historiens. Quelques articles sont trop courts : Bienfaiteurs et Entreprises (lien avec les) auraient gagné à être fusionnés. Un article manquant : "Ouest-France ", tant est fort le soutien de ce quotidien régional à l'enseignement catholique.

Un regret : l'ouvrage utilise le découpage régional de 1964, toujours en place. Certains seront chagrinés de l'exclusion de la Loire-Atlantique. D'autres estimeront que le cadre significatif aurait été un Grand Ouest intégrant tous les départements où l'enseignement catholique tient une forte place dans le second cycle (l'ancienne académie de Rennes plus les départements de la Manche et de l'Orne). Mais la Région Bretagne tenait sans doute à rappeler qu'elle a la charge des lycées. Un ouvrage qu'il ne faudrait pas réserver aux bibliothèques des lycées catholiques.

Jean-Luc MARAIS

BuRGuin, Pascal, Un lycée dans la guerre. Le lycée de garçons de Rennes, 1939-1945, préface d'Alain-François Lesacher, Rennes, Association pour la mémoire du lycée et du collège de Rennes (AmELYCOR)/Société archéologique et historique d'Illeet-Vilaine, 2017, 168 p.

L'ouvrage de Pascal Burguin se situe à la croisée de la recherche historique et de la démarche pédagogique, pour ne pas dire civique. À son origine, il y a un travail effectué avec différentes classes de Première du lycée Émile Zola dans le cadre du concours national de la Résistance et de la Déportation (CNRD) de 2009 à 2012 (le lycée de garçons de Rennes prend le nom de lycée Chateaubriand en 1960 puis de lycée Émile-Zola en 1971). Travail complété par les recherches de l'auteur aux Archives départementales d'Ille-et-Vilaine et aux Archives municipales de Rennes, ainsi que par les documents rassemblés depuis plusieurs années par l'AMELYCOR. Disons-le d'emblée, son aboutissement sous forme de livre est une réussite. Agrémenté de nombreuses illustrations, ce dernier nous renseigne sur la vie quotidienne d'un lycée durant la Seconde Guerre mondiale. Partant, il fait progresser la connaissance d'un sujet quelque peu délaissé par les chercheurs. En effet, alors que l'école primaire sous l'Occupation a récemment été analysée par Matthieu 